Classification

Physics Abstracts

$61.42+\mathrm{h}-61.16-\mathrm{d}$

\title{
Near field microscopy of glass surface and interfaces
}

\author{
François Creuzet
}

Laboratoire CNRS/Saint-Gobain “Surface du Verre et Interfaces”, 39 Quai Lucien Lefranc, 93303 Aubervilliers, France

(Reçu le 20 août 1993, accepté le 15 octobre 1993)

\begin{abstract}
Résumé. - Dans cet article, nous donnons une brève revue des activités de notre laboratoire dans le domaine des microscopies en champ proche. Tout d'abord, nous décrirons quelques résultats typiques obtenus avec un microscope à forces atomiques (AFM) sur la surface du verre et ses interfaces. La caractérisation de la rugosité, l'investigation des propriétés acido- basiques locales, la fracture des matériaux fragiles, l'adhésion de polymères et la croissance de couches métalliques sont autant d'exemples qui illustrent le potentiel de l'AFM pour l'étude des matériaux isolants. Ensuite, nous présenterons les principales spécifications que nous avons obtenues durant la phase de développement de deux nouveaux instruments: un microscope à forces atomiques fonctionnant sous ultra-vide et un microscope optique en champ proche pouvant donner accès à une spectroscopie locale dans le domaine infrarouge.
\end{abstract}

\begin{abstract}
In this paper, we will give a brief survey of the activity of our laboratory in the field of near field microscopy. Firstly, typical results obtained with Atomic Force Microscopy (AFM) on glass surface and interfaces will be described. Characterization of roughness, investigation of local acidbase properties, fracture of brittle materials, adhesion of polymers and growth mechanism of metallic layers are as many examples which illustrate the potential of AFM for the study of insulating materials. Secondly, we will present the main specifications that we have obtained during the development of new instruments: an Atomic Force Microscope working in ultra high vacuum (UHV) and a near field optical microscope with a spectroscopic capability in the infrared range.
\end{abstract}

\section{Introduction.}

In the glass industry, a large number of processes involve the properties of the surface: coatings with either metallic or organic or inorganic materials, reinforcement, corrosion, adhesion, friction, etc. Furthermore, silicate-based glasses might serve as characteristic materials for basic research on insulating surfaces, for instance as a model for fracture, an ideal substrate for thin films or organic layers, a surface for wetting studies. Because the relevant phenomena are more likely to take place at the atomic scale, near-field microscopes are expected to bring a new insight in these fields. In the case of insulators, atomic force microscopy (AFM) is the most efficient technique 
which can resolve details close to the atomic scale. All the results obtained in air or in liquid demonstrate that in most cases, the development of AFM in ultra high vacuum (UHV) is crucial for a better interpretation of the images. Photon Scanning Tunnelling Microscopy is also very promising, especially if a locally resolved spectrum can be acquired at different locations of the surface; we expect that a spatial resolution of about $1 \mu \mathrm{m}$ will be achieved in the infrared range.

\section{Experimental results obtained with AFM.}

The full description of Atomic Force Microscopy (AFM) will be found in the literature [1, 2]. In this paragraph, we will present results obtained with a commercial instrument from Park Scientific Instruments. When necessary, the capabilities have been slightly improved by using an external analog to digital converter.

2.1 RoughnESS. - Roughness is a spectral parameter and AFM can reveal details at short wavelength (typically within the range $1 \mathrm{~nm}-100 \mu \mathrm{m}$ ) which cannot be detected by other techniques. The information that is obtained at this scale is important either to characterize the manufacturing process or to analyze the modifications after surface treatment when glass is used as a substrate. Great care must be taken when roughness data are to be given: due to the digitization rate, the bandwidth is strongly dependent on the acquisition procedure. For instance, the analysis of AFM images given in reference [3] may prove successful in getting rid of systematic noise.

Another way to exploit the full capability of AFM is to record a single trace with more data points. Typically, a $10 \mu \mathrm{m}$ profile with 10000 points will give a rather complete set of data over the full range. Note that the digitization step must be kept close to the lateral resolution $(\approx 1 \mathrm{~nm})$ in order to avoid the aliasing effect. Figure 1 displays the traces that are obtained with bare float glass and $\mathrm{HCl}$ treated glass. The rms roughness of bare float glass is found to be close to $2 \AA$ and the histogram of the height distribution is well described as a Gaussian type with $\sigma=2.3 \AA$. A significant modification of roughness is found after chloric acid $(\mathrm{HCl})$ treatment of float glass. The histogram reveals that two Gaussian distributions (with $\sigma=1.8 \AA$ and $\sigma=5.7 \AA$, respectively) are necessary to account for the increase of the rms value. This indicates that the corrosion is inhomogeneous with the formation of pits. The visible peaks have a mean width of about 0.1 $\mu \mathrm{m}$. The drawback of this approach is that the appearance of the defects may be distorted; for example, if circular objects of a given diameter are randomly distributed at the surface, a single profile will show a large variety of entities, the width of which will range from almost zero up to the diameter of the circle.

2.2 ACID-BASE PROPERTIES. - AFM can be used to locally probe the acid-base properties of glass surface [4]. The method is based on the so-called double layer interaction [5] which takes place between two surfaces in an electrolyte. Each surface becomes charged after proton exchange with the electrolyte. The so-called double layer is made of the surface charges on the one hand, and the rearrangement of the ion concentration in the electrolyte on the other hand. The electrostatic potential due to the presence of a single surface exhibits an exponential decay ( $\Phi \propto$ $\exp (-r / \lambda)$ ) where $r$ is the distance to the surface and $\lambda$ is the Debye length (which is simply related to the ionic strength of the electrolyte [5]). When the two surfaces are close together, an electrostatic interaction results from the interpenetration of the two double layers. The force vs. distance profile is strongly dependent on the $\mathrm{pH}$ of the electrolyte: roughly, it crosses zero when $\mathrm{pH}$ is equal to the isoelectric point (IEP) of one of the two surfaces. In other words, when $\mathrm{pH}$ is equal to the IEP of one surface (there are as many $\mathrm{H}^{+}$sites as $\mathrm{OH}^{-}$sites on this surface), the net charge is zero and the electrostatic interaction vanishes. Figure 2 displays the force between the 

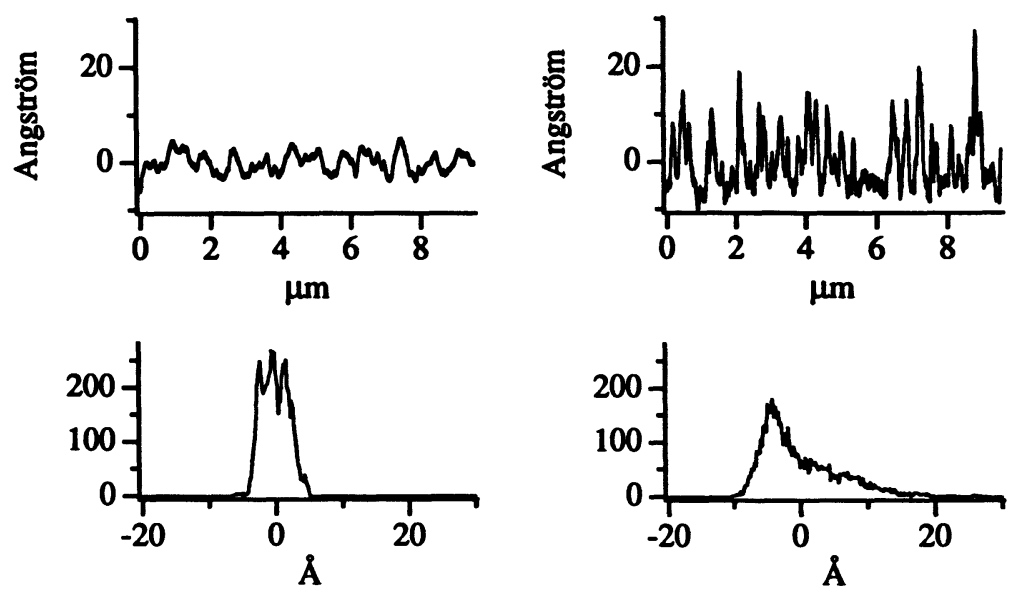

Fig. 1. - Roughness profiles and the corresponding histogram of bare float glass (left) and $\mathrm{HCl}$ treated glass (right).

$\mathrm{AFM} \mathrm{Si} \mathrm{N}_{3}$ tip and $\mathrm{SiO}_{2}$ surface as a function of the distance for different $\mathrm{pH}$ 's. A clear zero crossing occurs at $\mathrm{pH}=6.2$ which corresponds to the IEP of $\mathrm{Si}_{3} \mathrm{~N}_{4}$. This series of experiments has been carried out with an ionic strength of $10^{-3} \mathrm{~mol} / \mathrm{L}$ for the electrolyte. This value must be kept constant in order to compare results corresponding to a given Debye length. As a consequence, the $\mathrm{pH}$ values that could be investigated remain above 3 which exceeds the expected IEP of silica $(\approx 2)$. This is the reason why only one zero force crossing is observed here.

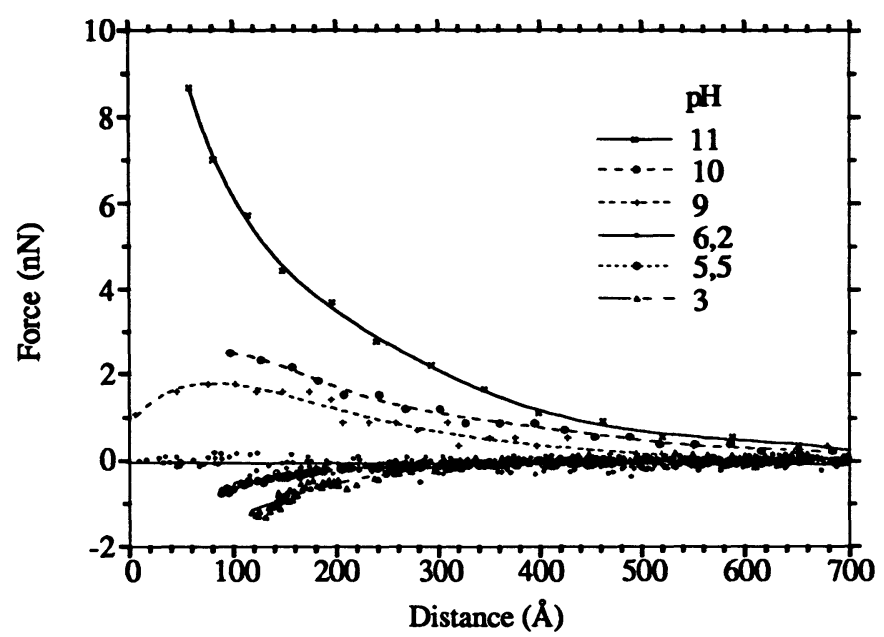

Fig. 2. - Double layer force as a function of the tip-silica distance at different pH's.

Based on the theoretical description of the double layer interaction, it is possible to give an estimate of the spatial resolution that may be obtained with this approach. It is approximated to $\sqrt{2 \pi R \lambda}$, where $\lambda$ is the Debye length and $R$ is the radius of curvature of the tip. This leads to a res- 
olution of about $400 \AA$. This will appear useful to probe flat surfaces which are not homogeneous with respect to IEP, either because of changes in the composition or because of local adsorption at the surface.

2.3 FRACTURE OF GLASS. - The theoretical models that have been developed to interpret the fracture of glass $[6,7]$ suffer from a lack of experimental evidences. Basically, the key phenomena take place at the surface on a very short scale (typically $1-10 \mathrm{~nm}$ ) and have never been observed so far. For instance, it is generally admitted that faults of about $10 \mathrm{~nm}$ wide are responsible for the large difference between experimental and theoretical rupture stress of glass fibers. We have undertaken the AFM observation of two different systems which have a well defined macroscopic behavior: aged glass fibers and indented flat glass.

The ageing procedure in a humid atmosphere is known to weaken the mechanical properties of glass fibers. AFM reveals the existence of rather large defects at the surface, the size of which may roughly account for the change of the rupture stress. The limitation of this approach is that the defects are statistically distributed on the fiber and it is difficult to decide which one prevails for fracture. Therefore, future work will focus on the preparation of controlled defects in the fiber; this should permit to define a model system which will be more easily compared to the theory.

Flaws can be prepared at the surface of glass by conventional Vickers indentation. We have imaged the far end of the crack tip after the load has been released. The end of the fault is roughly $15 \AA$ deep and $500 \AA$ wide and does not seem to depend on the nature of the glass (bare float glass, annealed float glass or silica). The far end is more like a hollow: the distance across is about $1500 \AA$ while the depth is reduced to $7 \AA$ or so. It remains difficult to reconcile this shape with any model found in the literature. We plan to study the modifications of this topography when the crack propagates in various environments (e.g. UHV, 100\% relative humidity, inert gas, etc.). This is aimed to study the role of intermolecular (van der Waals like) forces between the two edges of the flaw.

2.4 ADHESION OF POLYMERS. - As an example of glass-polymer interface, we will discuss the adhesion of polyesterurethane (PU) on float glass. Figure 3 is an image of a typical failure surface (substrate side) obtained after peeling of a $1 \mathrm{~mm}$ thick film of PU elastomer from a float glass substrate. In this case, no ageing of the assembly has been performed and the failure seems to be interfacial at the optical scale, i.e. occurring at the glass-polymer interface. Moreover, SEM observations predict that a smooth and homogeneous surface is to be found. On the contrary, small and randomly distributed islands of polymer residue are observed by AFM and two of them are shown on the top of the figure. Note that the rather small size of these islands may explain why they have not been detected by other techniques. Between the islands, the roughness is $6 \AA$ or so, a value unambiguously larger than the one of bare float glass; this indicates that an ultra thin residue of polymer covers the surface, in agreement with infrared spectroscopy results [8].

When the assembly is aged after a two-days annealing in a humid atmosphere, the peeling force decreases monotonously down to zero. The failure becomes cohesive, i.e. within the polymer itself, and polymer residue is observed on the whole surface [8]. However, the corrugation revealed by AFM indicates that the fracture of PU is inhomogeneous at the sub-micron scale: the surface consists of a multitude of bumps, more or less connected, and whose height is in the range 1000$4000 \AA$ and diameter is about $3000 \AA$. There is a close similarity between the shape of these bumps and the islands of figure 3: for the non aged assembly, the peaks inside the island are $\sim 200 \AA$ high and have a diameter distributed around $800 \AA$. A possible explanation is that the polymer tends to form fibrils during the peeling experiment: when the PU film is torn, these fibrils will eventually break and collapse to form the observed bumps. 


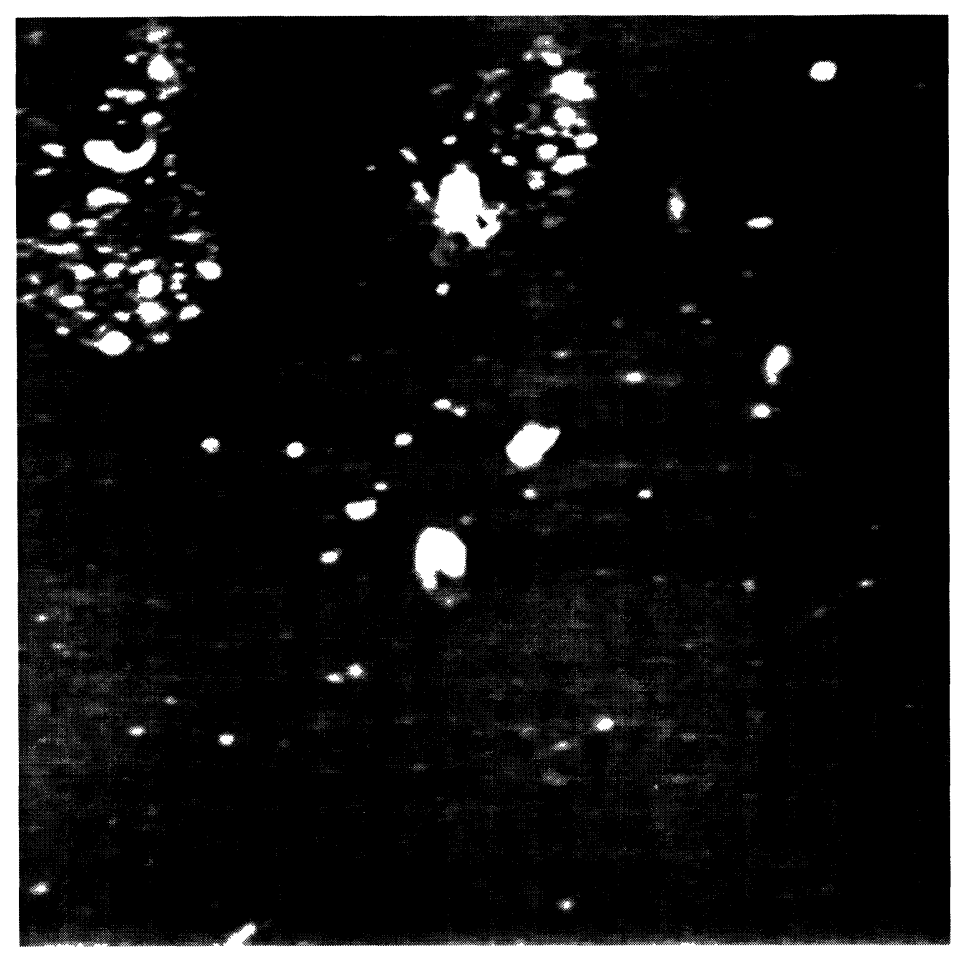

Fig. 3. $-7 \times 7(\mu \mathrm{m})^{2}$ AFM image of polymer residue after peeling a non-aged assembly.

These results can be interpreted within the interphase model [9] which takes into account the modification of the polymer in the vicinity of the interface. The thickness and the structure of this intermediate region, the so called "interphase", have a strong influence on the mechanical behavior of the assembly. In the case of PU/glass assemblies, the thickening of the interphase is believed to come from the alkaline character of glass, possibly because of diffusion of alkali ions from the float glass into the polymer during the annealing. Assuming that the failure takes place in the weakest part of the interphase, the AFM observations may be described as follows. For non aged assemblies, the interphase is so thin that the failure is almost interfacial: the residue is mainly made up of a thin film of PU, except when some kinc of impurity or surface inhomogeneity has weakened the PU or reinforced the interface at some places (islands in Fig. 3). More work is necessary to characterize the nature of these defects, especially to precise the possible role of contamination and charging effects. In the case of a 2-days aged assembly, the failure is clearly cohesive, but large bumps are observed which suggest an organization of the polymer during the peeling test. Note that in all cases, the structure of the peeled surface is heterogeneous at a scale of 100 to $1000 \AA$. More information on this subject may be found in reference [10].

2.5 GROWTH MECHANISM OF METALLIC LAYERS. - The growth mechanism of metal thin films on oxides and the adhesion of the final coating are believed to be strongly correlated. In most cases, the relevant interaction could be non-reactive in the sense that no chemical reaction takes place at the interface. The observation of the surface at the first stages of the growth is expected to be an important experiment for identifying the pertinent parameters. Figure 4 shows the AFM 
observation of a very thin (average thickness of about $10 \AA$ ) -far below percolation- silver deposit on float glass. Well-defined aggregates of $500-1000 \AA$ wide and $15 \AA$ thick are observed. These characteristics might be due to the sputtering technique used for this deposition since such large islands are not observed when the metal is directly evaporated on the substrate. It is worth noticing the good wetting of the metal on the glass substrate. Theoretical work based on the dielectric properties of the materials is under progress in our laboratory to try to explain this surprising behavior [11].

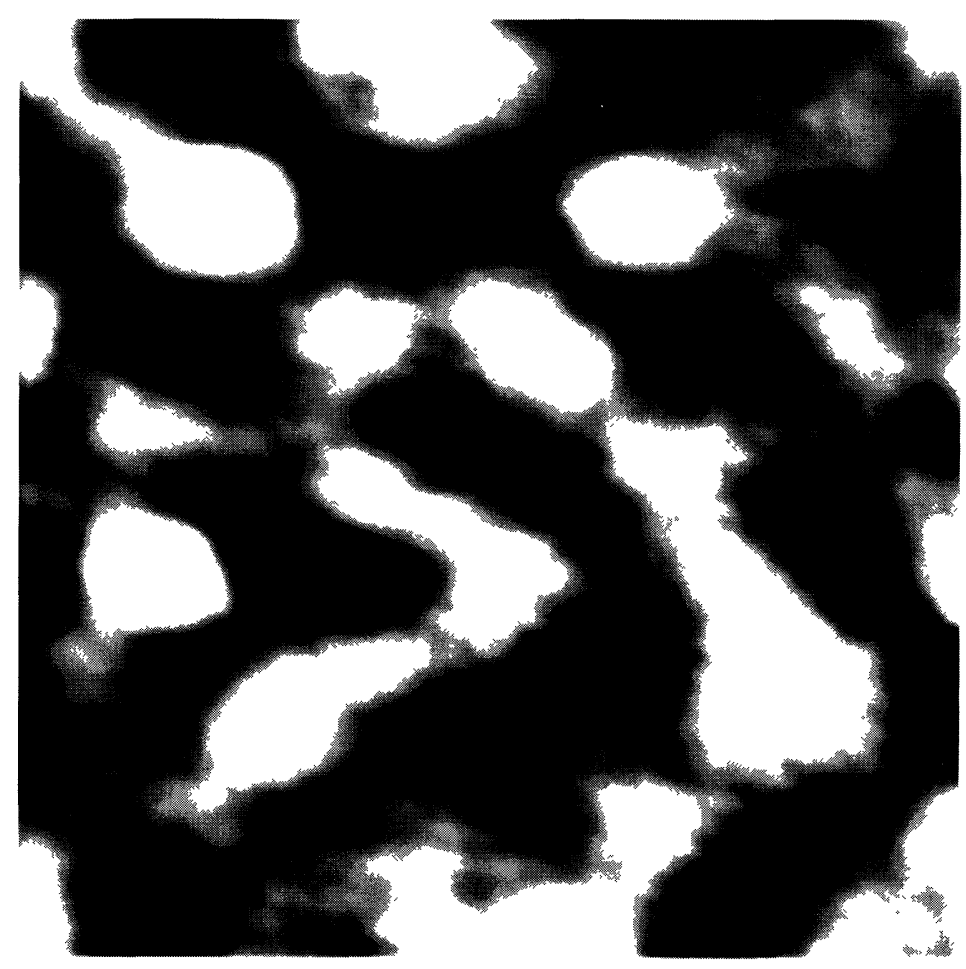

Fig. 4. $-300 \times 300(\mathrm{~nm})^{2}$ AFM image of a very thin silver deposit on float glass. The gray scale corresponds to $6.8 \mathrm{~nm}$.

\section{Development of new instruments.}

Our work with AFM on glass shows that two problems must be circumvented if surface science on insulating materials is to be done. First, it is necessary to reproducibly control the environment and the surface contamination. This will be achieved by working in Ultra High Vacuum (UHV). Second, it would be very useful to have a chemical signature of the objects that we observe with AFM. Our work in this direction is to build a Photon Scanning Tunnelling Microscope working in the infrared range. 
3.1 ATOMIC FORCE MICROSCOPY IN ULTRA HIGH VACUUM. - The AFM microscope that has been developed in our laboratory is based on the beam deflection detection technique. The light is guided into the UHV chamber by focusing a laser beam on an optical fiber. A one stage spring system and a magnetic damping ensure the proper reduction of mechanical vibrations. Typical vacuum which can be obtained is of the order of $10^{-10}$ Torr. So far, we have tested our prototype by imaging the mica surface: the well-known hexagonal lattice is observed with good contrast and stability. Roughly, the specifications are similar to those of commercial instruments. The UHV measuring chamber is connected to a preparation chamber with the following capabilities: sample cleaning, sputtering technique, XPS analysis.

3.2 PHOTON SCANNING TUNNELLING MICROSCOPY WORKING IN THE INFRARED RANGE. - We can illustrate the expected applications of this microscope by discussing the example of polymer adhesion given above. Figure 3 shows heterogeneities which cannot be chemically characterized; if the islands are likely to be polyurethane, it is difficult to decide if the organic matter in between is of the same origin or if it is broken chains. Obviously, a local infrared (IR) spectroscopy would permit to answer this question. Therefore, we have undertaken the development of a microscope which will permit to image the surface and subsequently to acquire an IR spectrum at a welldefined location. The specifications that are desirable for this locally resolved spectroscopy are the following: lateral resolution in the $1 \mu \mathrm{m}$ range, spectral resolution of about $10 \mathrm{~cm}^{-1}$ over the spectral window [ $1000-10000 \mathrm{~cm}^{-1}$ ] and a signal to noise ratio of 1000 in order to detect the IR absorption signal of a $10 \mathrm{~nm}$ layer.

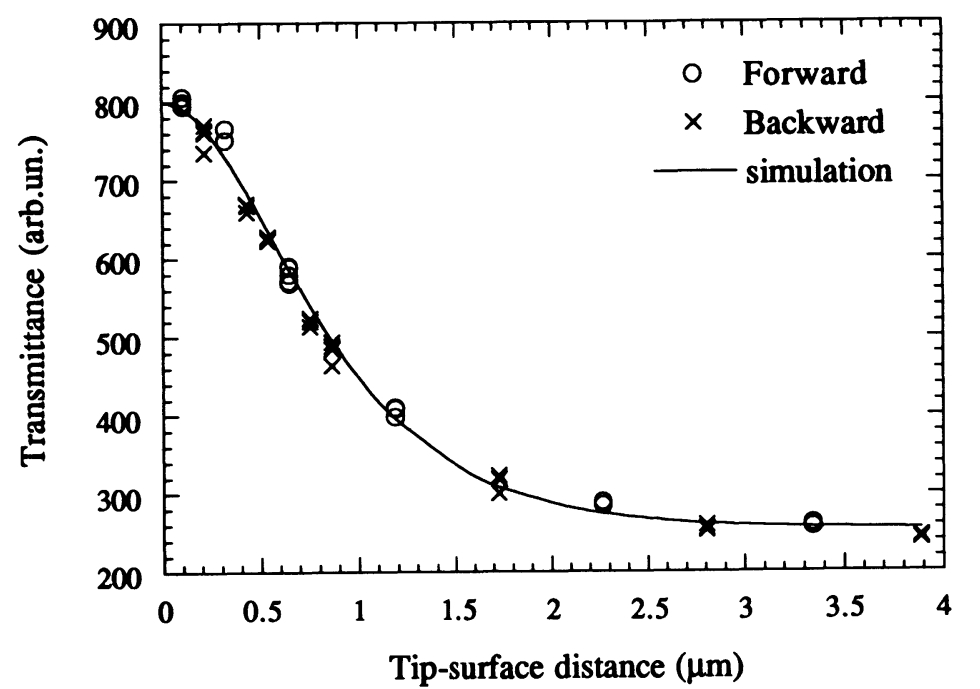

Fig. 5. - Decay of the evanescent field recorded at a wavelength of $5 \mu \mathrm{m}$.

The experimental set-up is based on the total internal reflection arrangement which gives the best lateral resolution in the visible range [12]. So far, we have used two infrared sources: a conventional Globar source (which may be approximated to a blackbody at $1300 \mathrm{~K}$ ) and a tuneable IR laser, the so-called CLIO source, which is a free electron laser operated at LURE (Orsay) [13]. We routinely detect the evanescent field, an example of which is given in figure 5 in the case of 
a $5 \mu \mathrm{m}$ wavelength. Comparison with theoretical calculations [14] is satisfactorily achieved for both forward and backward data. More details can be found in reference [15] which compares the results obtained with the two different sources. This characteristic behavior is the key part of the photon scanning tunnelling microscopy and can be recorded with fibers having a radius of curvature as small as $1 \mu \mathrm{m}$.

Preliminary results on spectroscopy are very promising. The characteristic doublet of a thin layer $(\approx 0.6 \mu \mathrm{m})$ of a photosensitive resin has been recorded in the near field regime by stepping the CLIO wavelength through the IR band of interest. Details can be found in reference [16]. Because of the rather low power per area unit, these latter data have been acquired with a large $(\approx$ $10 \mu \mathrm{m}$ in diameter) fiber tip. In order to obtain the specifications given above, we will implement another source, a cascade arc, which will permit to increase the signal by about two orders of magnitude.

\section{Acknowledgements.}

Most of the results presented in this review have been obtained in collaboration with other members of the laboratory: D. Abriou, H. Arribart, F. Didier, E. Guilloteau, C. Jolu, X.Y. Lin, A. Piednoir and G. Ryschenkow.

\section{References}

[1] Binnig, G. Quate C.F., Gerber Ch., Phys. Rev. Lett. 56 (1986) 930.

[2] Rugar D., Hansma P., Physics today (October 1990) p. 23.

[3] Dumas Ph., Bouffakhreddine B., Amra C., Vatel O., Andre E., Galindo R., Salvan F., Europhys. Lett. 22 (1993) 717.

[4] Lin X.Y., Creuzet F., Arribart H., J. Phys. Chem. 97 (1993) 7272.

[5] Israelachvilii J.N., Intermolecular and Surface Forces: with Applications to Colloidal and Biological Systems (2nd edition, Academic Press, 1989).

[6] Dugdale S., J. Mech. Phys. Solids 8 (1960) 100;

Barenblatt G.I., Adv. Appl. Mech. 7 (1962) 55.

[7] Griffith A.A., Proc. First. Int. Congress of Appl. Mechanics, Biezeno C.B. and Burgers J.M. Eds., Delft (1924) p. 55.

[8] Berquier J.M., Buffeteau T., Chartier P., Desbat B., Arribart H., C.R. Acad. Sci. Paris, t. 315 (1992) p. 1623.

[9] Sharpe L.H., Proc. of the 162nd Meeting of the American Chemical Society, Div. Org. Coat., Plast. Chem. Preprints 31 (1971) 201.

[10] Creuzet F., Ryschenkow G, Arribart H., J. Adhes. 40 (1992) 15.

[11] Didier F., Jupille J., submitted to Surf. Interf. Anal, Proceedings of ECASIA'93, Catania, Italy.

[12] Reddick R.C., Warmack R.J., Ferrell T.L., Phys. Rev. B 39 (1989) 767.

[13] Ortéga J.M., Billardon M, Pour la Science (April 1986) 91.

Glotin F., Chaput R., Jaroszynski D., Prazeres R., Ortéga J.M., Phys. Rev. Lett. 71 (1993) 2587.

[14] Salomon L., Thesis, Université de Bourgogne (Dijon), February 1991;

Zhu S., Yu A.W., Hawley D., Roy R., Am. J. Phys. 54 (1986) 601.

[15] Piednoir A., Creuzet F., Licoppe, De Fornel F., Near Field Optics, D.W. Pohl and D. Courjon Eds. (Kluwer Academic Publishers, 1993) p. 309.

[16] Piednoir A., Creuzet F., Licoppe C, Ortéga J.M., Proceedings of the Near Field Optics conference, Raleigh (U.S.A.) October 1993. 